\title{
Review on active suspension system
}

\author{
Aizuddin Fahmi Mohd Riduan ${ }^{1}$, Noreffendy Tamaldin ${ }^{2}$, Ajat Sudrajat ${ }^{3}$, and Fauzi \\ $\mathrm{Ahmad}^{4}$ \\ ${ }^{1,2,4}$ Centre for Advanced Research on Energy, Universiti Teknikal Malaysia Melaka, Hang Tuah \\ Jaya, 76100 Durian Tunggal, Melaka Malaysia. \\ ${ }^{1,2,4}$ Faculty of Mechanical Engineering, Universiti Teknikal Malaysia Melaka, Hang Tuah Jaya, \\ 76100 Durian Tunggal, Melaka, Malaysia. \\ ${ }^{3}$ Engineering Physics, Faculty of Science and Technology, Universitas Nasional-Jakarta JL. Sawo \\ Manila, Pasar Minggu, Jakarta 12520, Indonesia.
}

\begin{abstract}
For the past decade, active suspension systems had made up most of research area concerning vehicle dynamics. For this review, recent studies on automobile active suspensions systems were examined. Several vehicular suspension types were also described to compare amongst them. From published investigations by previous researchers, various automotive suspensions in terms of cost, weight, structure, reliability, ride comfortability, dynamic and handling performance were exhibited and compared. After careful examination, it was concluded that electromagnetic active suspensions should be the general direction of vehicle suspension designs due to its energy regeneration, high bandwidth, simpler structure, flexible and accurate force control, better handling performance as well as drive characteristics.
\end{abstract}

Keywords: Active Suspension, Handling Performance, Dynamic Performance

\section{Introduction}

Vehicle suspension main task is to separate passenger and vehicular body interactions from oscillations generated by road abnormalities whilst still maintaining continuous wheel-road contact. Currently, there are three types of automotive suspensions commonly used namely passive, semi-active, and active.

All said systems are based by either pneumatic or hydraulic operation. It was asserted that some of these suspension systems cannot fully solve automobile oscillations problem because they are very costly and lend towards vehicle energy consumption increment [1].

Active vehicle suspension systems had been in wide investigation for more than 20 years due to their promising characteristics. These systems poses potentiality of responding considerably good towards upward and downward changes caused by road inputs irregularities since the dampers as well as springs are mediated by an actuator force. Actuator in an active suspension functions to spread energy away from system and it can be operated through various kinds of controllers determined by proposed design. With correct managing techniques which could induce more beneficial compromise between driving 
comfort and road handling stability, an active system as a whole would be the superior suspension design [2].

Sophisticated electromechanical and electronic systems are used to affect automobile dynamic performances such as anti-lock braking system (ABS), electronic stability control (ESC), electronic brake force distribution, and many more. These systems advancement towards supporting passenger safety and vehicle handling have become an increasing requirement in the automotive industry, especially when vehicles nowadays can vary in design scopes from either being small, having higher centre of gravity, or aim to be environmental friendly.

One other present trend in the automotive industry is movement towards fully electric vehicles. These cars possess different overall weight distribution due to an internal combustion engine (ICE) is substituted with an electric motor and a battery pack that weighs more or less $400 \mathrm{~kg}$. Efficiency of electrical drive train is best optimized when it is fully incorporated to the wheel. Even so, car unsprung ass will gain which could cause problems with respect to passenger comfort and handling. Hence, this clearly shows the need for active suspension be integrated in automobiles [3].

Automotive manufacturers often compete against each other to develop dependable and comfortable drive characteristics while at the same time also producing best stability during braking and cornering. With passive suspension however, it could just provide one of the stated features or at most compromise between handling and ride comfortability. Nevertheless, selected companies do offer adjustable suspension system options which can be tuned according driver comfortability needs. Because of this, researchers had proposed and employed several semi-active and active vehicle suspension systems both in theoretical and experimental terms [4]. These systems main benefit is that they simultaneously permits eliminating most road interferences from pitch control as well as active roll.

\section{Classifications of automotive suspensions}

\subsection{Passive Suspension}

Passive vehicle suspension systems modelled in Fig. $\mathbf{1}$ is a typical mechanical suspension arrangements comprising viscous damper and linear springs with constant damping coefficient and stiffness respectively [2].

Passive vehicle suspension is reliable, not complex, as well as inexpensive. The damper and spring in this system are fastened between car body and wheel support structure. A damper insides is occupied with a hydraulic oil or compressed gas, and there is a piston moving by a rod from its exterior. Piston movement is permitted by a hole that allows fluid passing among cylinder parts. This flow of fluid develops a reactional force which is relative towards proportional speed between unsprung and sprung masses. Damping is then attained by converting oscillations energy into heat.

This uncomplicated system cannot yield decent outcomes for counteracting suspension problems since it does not give any external control and feasible betterments in terms of materials, hole valves, or even shapes [1]. 


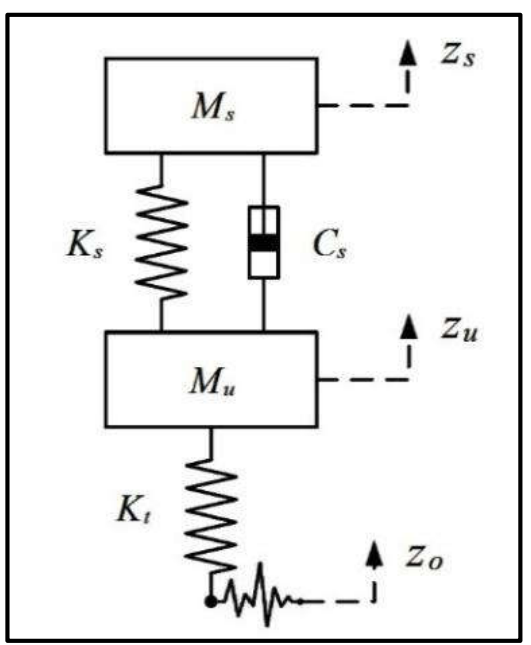

Fig. 1. Passive suspension model [6]

\subsection{Semi-active Suspensions}

Semi-active suspension is similar to a passive design with only difference being having a variable damping coefficient but still fixed spring constant and also absence in active force sources. This kind of system allows seamless change between a passive damper with semiactive damping coefficient as shown in Fig. 2.

While maintaining fixed spring utilization, the employed continuous variable damper alters workability in real time through closed feedback loop control design to assure proper energy dissipation, thus bettering suspension overall performance against a passive system [5].

A semi-active suspension can be remotely switched electrically to stiffen or soften the system making its damping coefficient either varies discontinuously or continuously. Shifting in functioning strategies like stiffening suspension would benefit vehicle at cornering, braking, acceleration, reducing low frequency response towards inertial forces associated with roll and pitch, as well as avoiding wheel resonances and body excitation.

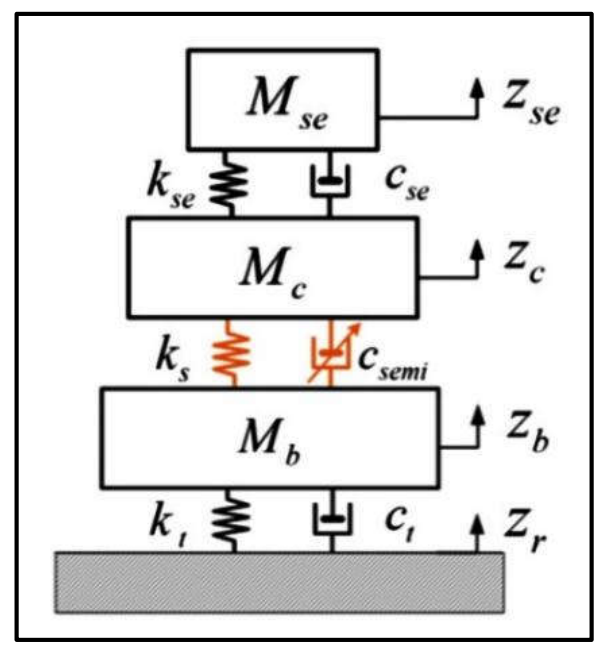

Fig. 2. Semi-active model [7]

\subsection{Active Suspensions}

Compared to other previous systems, an active suspension includes an actuator that can 
supply active force regulated by a controlling algorithm which uses information gathered from attached vehicle sensors. As illustrated in Fig. 3, active suspension comprises an actuator, mechanical spring, and damper; or an actuator and mechanical spring only.

These kind of systems have much better reacting capabilities against generated vertical forces caused by unpredictable road input changes since the dampers as well as springs are regulated through an actuator force. This actuator operates by allowing or spreading energy from the system and could moderate through different controller types based on intended design. With proper controlling methods, an active suspension can result in compromise between vehicle ride comforts to road handling stableness be more improved, thus making it an overall enhanced suspension design.

Because of this, most researchers recognized that this system type are among best ways in improving its overall performance due to the ability in managing contradictory parameters [5]. Even so, it is a complicated and expensive system which explains this particular system usage by only a small number of high-end car models, or trucks [1].

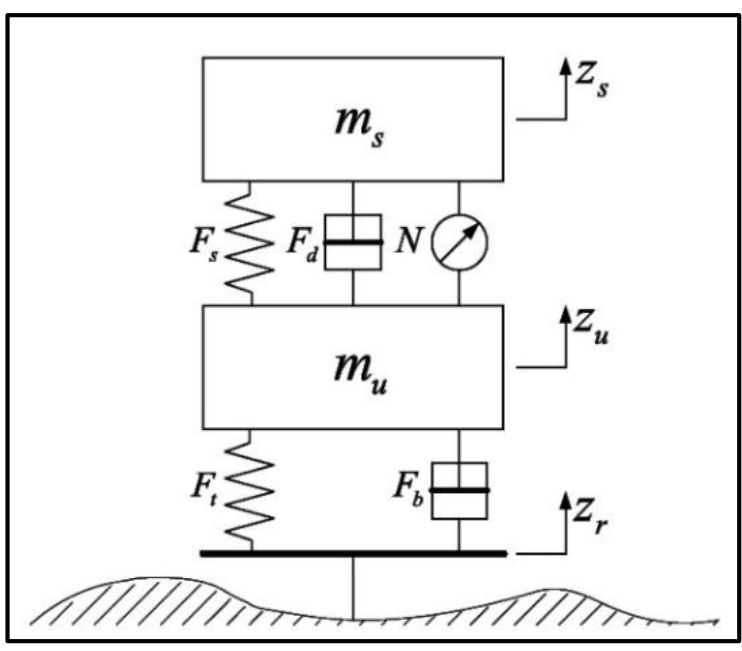

Fig. 3. Active model $[8]$

\section{Hydraulic or pneumatic active suspensions}

Active suspension system with pneumatic or hydraulic actuator are managed through electric drives and the power is provided from a battery source or conventional ICE itself. Typically, hydraulic systems are more widely used in body control systems due to its design simplicity, high force density, technology maturity, reliability, as well as various commercial parts availability.

For example, BMW recently developed an anti-roll control system by putting a hydraulic rotary actuator at rear of vehicle anti-roll bar centre. Another instance was exemplified by Mercedes active body control system which applies high pressure hydraulics to pre-stressing the springs, therefore developing anti-roll forces without coupling right and left wheels [2]. However, there are disadvantages in using a hydraulic system such as:

a. Inefficiently performed because continuously requiring a pressurized system

b. Comparatively high system time constant (flexible hoses and pressure loss)

c. Environmentally damaging due to toxic hydraulic fluids presence caused by hose leaks and ruptures

d. System volume and infeasibility space requirements even though it primarily contribute towards overall sprung mass 


\section{Electromagnetic active suspensions}

An electromagnetic active suspension system consists a spring and electromagnetic actuator setup in parallel arrangements within unsprung and sprung mass. The electromagnetic actuator performs with regards to electric supplied range by implemented control systems. It brings about active controlled forces for speedily absorbing road shocks, suppressing pitch and roll motions, and improving comfort as well as safety. Bi-directional amplifier electromagnetic actuator on the other hand, works by yielding and reducing electric power consumption compared against a hydraulic actuator [2]. After comparing with hydraulic actuators, electromagnetic actuators main superiority are as bellow:
a. Efficiency increment
b. Dynamic behaviour improvement
c. Stability betterment
d. Force control precision
e. Dual actuator operation

The weak points meanwhile are:

a. Suspension volume increment

b. Relatively greater current requirement from a 12 to $14 \mathrm{~V}$ system

c. Conventional designs which requires excitation to supply continual force

d. More expensive of a system

\section{Comparisons}

Table 1 below summarized suspension system comparisons with respect to descriptive classification of reviewed vehicular suspensions.

Table 1. Comparison between various automotive suspension systems

\begin{tabular}{|l|c|c|c|c|}
\hline \multicolumn{1}{|c|}{ Parameters } & $\begin{array}{c}\text { Passive } \\
\text { suspensions }\end{array}$ & $\begin{array}{c}\text { Semi-active } \\
\text { suspensions }\end{array}$ & $\begin{array}{c}\text { Hydraulic/pne } \\
\text { umatic }\end{array}$ & $\begin{array}{c}\text { Electromagnetic } \\
\text { active }\end{array}$ \\
\hline Structure & Simplest & Complex & Most Complex & Simple \\
\hline Weight/Volume & Lowest & Low & High & Highest \\
\hline Cost & Lowest & Low & Highest & High \\
\hline Ride comfort & Bad & Medium & Good & Best \\
\hline Handling & Bad & Medium & Good & Best \\
\hline Reliability & Highest & High & Medium & High \\
\hline Dynamic & Passive & Passive & Medium & Good \\
\hline
\end{tabular}

\section{Recent innovations on suspension}

\subsection{Magnetic dampers}

By altering electric current through a ferrous fluid, Magnetic Ride Control (MRC) dampers can adapt its stiffness in response towards specific driving situations. Even though this system is licensed to other car manufacturers, General Motors (GM) which first designed it is still leading in competition. Its third generation MRC as shown in selected performance vehicles like the Chevrolet Corvette Stingray (Fig. 4), amended former designs with a 
second wire addition. With that component add-on, suspension fluid could interchanged from firm to standard mode actively, whereas previously lag would be present when particles regained back to their normal state.

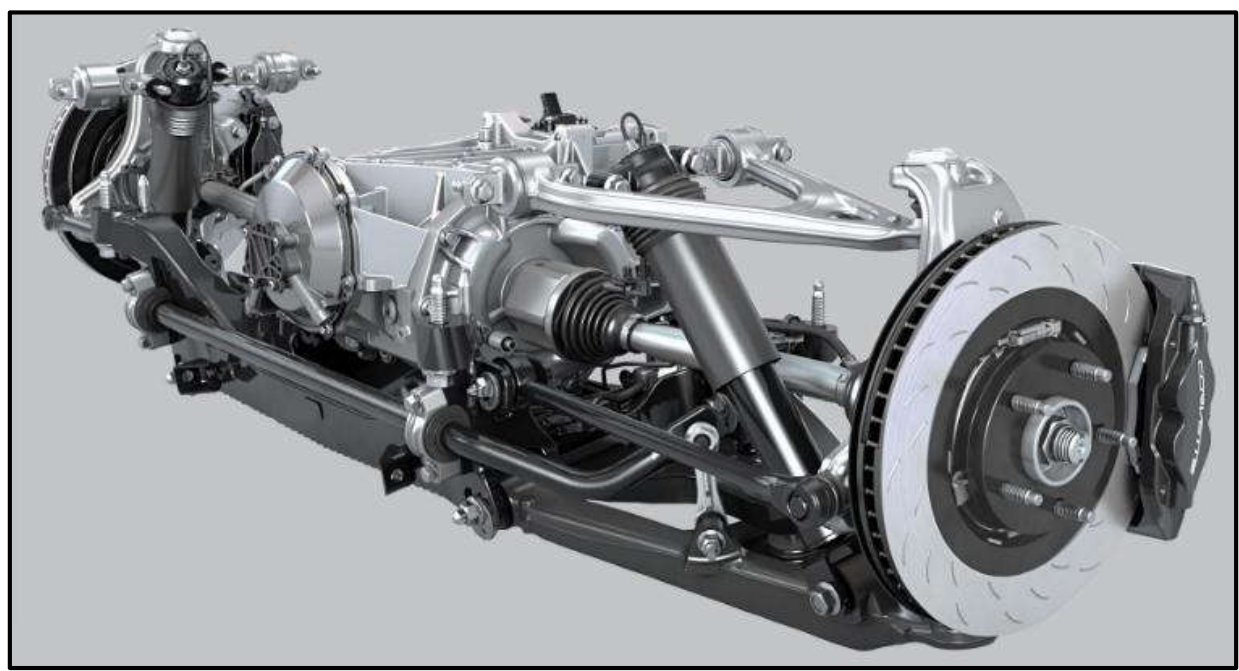

Fig. 4. Electromagnetic suspension [9]

\subsection{Active Curve Tilting}

This technology was developed in 2015 by Mercedes-Benz and its implementation in the S65 AMG Coupe utilizes a lateral acceleration sensor paired with a forward looking camera to perceive corners whilst also employing air suspension to tilt its body in towards the apex. As displayed in Fig. 5, this application was not aim for increasing cornering performance but instead enhancing ride comfortableness as passenger would undergo lower lateral loads at a given speed. Essentially, if road surface does not have a well banked corner, the vehicle would simulate one for its occupants.

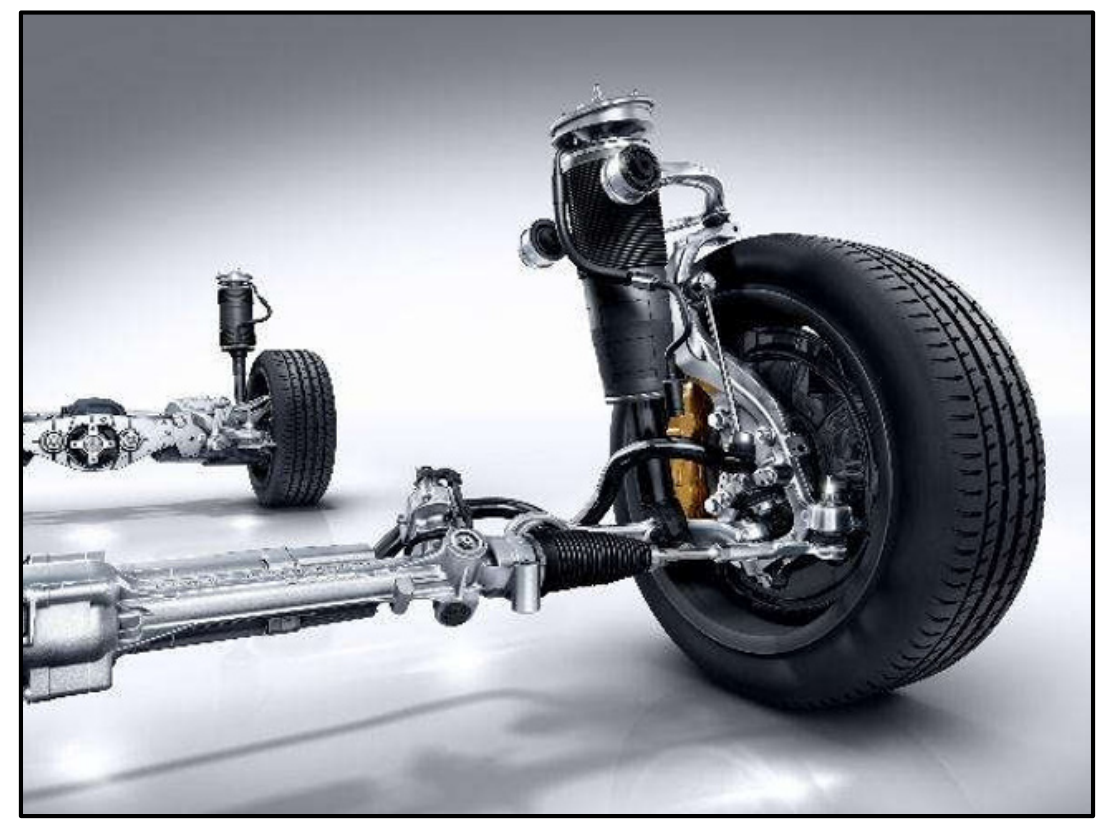

Fig. 5. Active Curve Tilting suspension [10] 


\subsection{Hydraulic Roll Control}

Audi RS 5 shown in Fig. 6 is one of the recent automobiles to feature a hydraulic crosslinked suspension which Audi designated as Dynamic Ride Control. Unlike mainstream suspensions which applied steel anti-roll bars for counteracting body roll, this hydraulic system would transfer fluid towards the vehicle opposite side. It is as if possessing a huge anti-roll bar but without any at all, depending on driving conditions. Cars with best balance between handling and performance would to some extent use variations of this approach. Even though hydraulic cross-linking is not entirely perfect, but it is currently the nearest suspension technological design with regards to a fully active suspension.

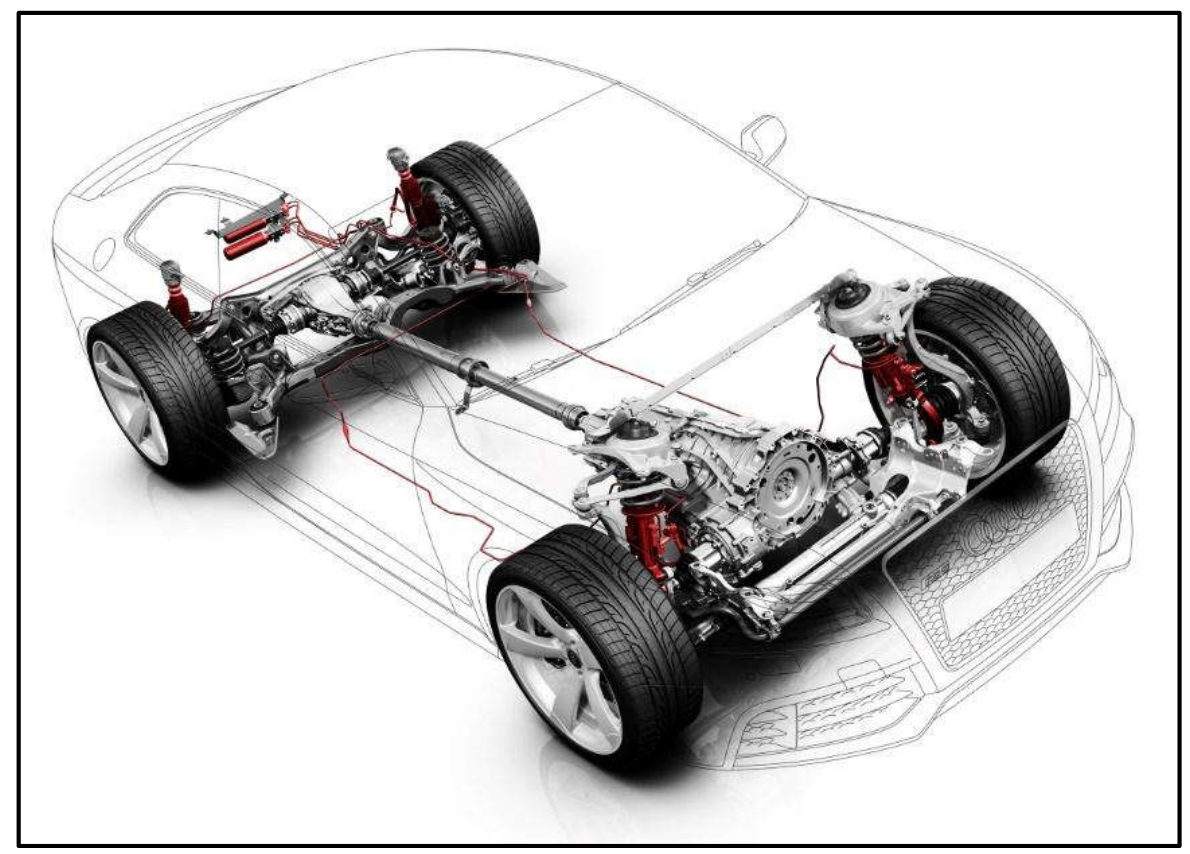

Fig. 6. Hydraulic Roll Control suspension [11]

\section{Conclusions}

Published work concerning on vehicle suspensions studies and its recent developments had been looked upon thoroughly in this paper.

Based on examined findings, electromagnetic active suspensions was postulated to be the future tendency of automotive suspension designs due to its energy regeneration, not complex structure, high bandwidth function, flexible and accurate force control, good ride quality as well as handling performance.

In recent years, advancement in power electronics, microelectronics, and permanent magnet materials had permitted significant betterments in electrical drives domain.

Steady and dynamic state performance, weight and volume decrement, electronic control system unconstrained incorporation in cars, reliability, as well as downsizing are crucial factors for rationalizing towards more generalized electrical drive utilizations.

The progression in this technology field warrants analysis of possibility enforcing available suspension systems with electromagnetic actuators to increase its performance without raising costs and energy consumption.

Succeeding investigations in electromagnetic active suspensions should concentrate on two main prospects which are research and developing more towards electromagnetic linear actuators as well as better management of the system. 


\section{Acknowledgement}

The authors would like to acknowledge Center of Advance Research on Energy (CARe), Universiti Teknikal Malaysia Melaka (UTeM) and Engineering Physics Department, Faculty of Science and Technology, Universitas Nasional-Jakarta for their continuous support in this study.

\section{References}

1. I. Martins, J. Esteves, F. P. da Silva, P. Verdelho, Electromagnetics hybrid activepassive vehicle suspension system, Technical University of Lisbon, Lisbon, Portugal (2015)

2. M. Mohammed Bello, A. Y. Babawuro, S. Fatai, Active suspension force control with electro-hydrolic actuator dynamics, ARPN Journal of Engineering and Applied Sciences, 10, 23, pp.17327 - 17331 (2015)

3. B. L. J. Gysen, J. L.G. Janssen, Active Electromagnetic Suspension System for Improved Vehicle Dynamics, IEEE Transactions On Vehicular Technology, 59, 3, pp.1156 - 1163 (2016)

4. R. Rosli, M. Mailah, G. Priyandoko, Active Suspension System for Passenger Vehicle using Active Force Control with Iterative Learning Algorithm, WSEAS Transactions on Systems and Control, 9, 2, pp.120 - 127 (2014)

5. M. Shafie, M. Bellob, R. M. Khan, Active Vehicle Suspension Control using Electro Hydraulic Actuator on Rough Road Terrain, Journal of Advanced Research in Applied Mechanics, 9, 1, pp.15-30 (2015)

6. M.M. Moheyeldein, A.M.A. El-Tawwab, K.A.A. El-gwwad, M.M.M. Salem, An analytical study of the performance indices of air spring suspensions over the passive suspension, Beni-Suef University Journal of Basic and Applied Sciences (to be published)

7. K. Sim, H. Lee, J.W. Yoon, C. Choi, S.H. Hwang, Effectiveness evaluation of hydropneumatic and semi-active cab suspension for the improvement of ride comfort of agricultural tractors, Journal of Terramechanics, 69, pp. 23 - 32 (2017)

8. H. Pan, W. Sun, X. Jing, H. Gao, J. Yao, Adaptive tracking control for active suspension systems with non-ideal actuators, Journal of Sound and Vibration, 399, pp. $2-20$ (2017)

9. Motor Trend: 2014 Chevrolet Corvette Stingray Z51 First Test. [online] Available at: https://www.motortrend.ca/en/news/2014-chevrolet-corvette-stingray-z51-firsttest/\#2014-chevrolet-corvette-stingray-z51-suspension [Retrieved $1^{\text {st }}$ August 2018]

10. Popular Mechanics: 3 Technologies That Are Making Car Suspensions Smarter Than Ever. [online] Available at: https://www.popularmechanics.com/cars/car-technology/a14665/why-car-suspensionsare-better-than-ever/ [Retrieved $1^{\text {st }}$ August 2018]

11. Audi Technology Portal: Dynamic Ride Control. [online] Available at: https://www.audi-technology-portal.de/en/chassis/suspension-controlsystems/dynamic-ride-control_en [Retrieved $1^{\text {st }}$ August 2018] 